\title{
Effectiveness and Safety of Plum - Blossom Needle in the Treatment of Myopia区A Protocol for Systematic Review and Meta-analysis
}

\section{Siyuan Zhu}

Jiangxi University of Traditional Chinese Medicine

Jun Xiong ( $\nabla$ xiongjun196071@163.com )

Affiliated Hospital of Jiangxi University of TCM https://orcid.org/0000-0002-4196-3351

Jun Chen

Jiangxi University of Traditional Chinese Medicine

Genhua Tang

Jiangxi University of Traditional Chinese Medicine

Lunbin Lu

Jiangxi University of Traditional Chinese Medicine

Zhiying Zhong

Jiangxi University of Traditional Chinese Medicine

Xingchen Zhou

Jiangxi University of Traditional Chinese Medicine

Han Guo

Jiangxi University of Traditional Chinese Medicine

Hao Fan

Jiangxi University of Traditional Chinese Medicine

\section{Protocol}

Keywords: plum-blossom needle, adolescent, myopia, protocol, systematic review $₫$ meta-analysis

Posted Date: September 9th, 2020

DOI: https://doi.org/10.21203/rs.3.rs-73070/v1

License: (c) (i) This work is licensed under a Creative Commons Attribution 4.0 International License.

Read Full License 


\section{Abstract}

\section{Background:}

In recent years, the prevalence of myopia has increased significantly, and it has become one of the major eye diseases that cause visual impairment in the world,which is particularly prominent among young people. And uncorrected myopia is the leading cause of blindness. The purpose of this study is to evaluate the efficacy and safety of plum-blossom needle in delaying adolescent myopia progression through systematic evaluation.

\section{Methods and analysis:}

The following electronic databases will be searched from inception to July 2020 regardless of publication status and language: Medline, EMBASE, Web of Science, the Cochrane Library, PubMed, China National Knowledge Infrastructure (CNKI), China Biology Medicine (CBM), Chinese Scientific Journal Database (VIP), Wanfang Database, Chinese Biomedical Literature Database (CBLD), Chinese Science and Technology Periodical Database (CSTPD). RCT registration websites, including http://www.ClinicalTrials.gov and http://www.chictr.org.cn, will also be searched. Review Manager V.5.4 will be used to analysis the statistic. Two reviewers will independently select studies, extract and code the data, assess risk of bias of the included studies, evaluate the quality of evidence for outcomes.

\section{Discussion:}

So far, many studies have been conducted on the treatment of adolescent myopia with plum-blossom needles. However, there is still no clear conclusion on the effectiveness and safety of plum-blossom needles in the treatment of juvenile myopia.In this systematic review and meta-analysis, available data will be pooled together to further inform research and clinical practice.

\section{Systematic review registration: Inplasy protocol 202080026}

\section{Background}

Myopia is a type of refractive error.When the eye is in a relaxed state, parallel light enters the eye and focuses in front of the retina, which results in the inability to form a clear image on the retina, which is called myopia. ${ }^{[1]}$ In the past few decades, the incidence of myopia has been increasing, and it has become one of the main diseases that cause visual impairment in the world. ${ }^{[2,3,4]}$ At present, $30 \%-50 \%$ of the population in the United States and Europe suffer from myopia, and this condition is more common in East Asia, where up to $90 \%$ of adolescents suffer from myopia. ${ }^{[2,3,5]}$ A study predicts that by 2050 , there will be $49.8 \%$ of the global myopia population (475.8 million people) and $9.8 \%$ of the highly myopic population (938 million people). ${ }^{[4]}$ At the same time, myopia is not only a refractive error, but also a visionthreatening disease. ${ }^{[6]}$ As the degree of myopia deepens, related complications such as cataracts, glaucoma, subretinal neovascularization, macular degeneration and so on will lead to severe visual 
impairment and even blindness. ${ }^{[7]}$ And globally, a common cause of impaired vision is myopia, and uncorrected myopia is the leading cause of blindness. ${ }^{[4]}$ In recent years, the increase in myopia rate seems to be closely related to the widespread popularization of electronic screen products, increasing educational pressure, and lifestyle changes that reduce the time young people spend outdoors, ${ }^{[7]}$ and myopia presents a trend of high morbidity and younger age, which seriously affects the physical and mental health of children.

Teenagers are at an important stage of learning, and long-term study at the desk can lead to long-term eyesight in a narrow near vision environment. At the same time, the eyeballs of this age group are in the stage of growth and development, and the refractive state is not yet completely stable. The theory of accommodation believes that long-term excessive tension of the ciliary muscle can cause accommodative myopia. If it can not be eliminated in time and sustained development, it will affect the normal progress of the metabolic function of the eye tissues, and the anterior and posterior diameter of the eyeball will gradually become passively elongated and become true myopia. Therefore, if active intervention and treatment can be carried out during the susceptible period of adolescent myopia, it will have important clinical significance for its prevention and treatment. ${ }^{[8]}$

At present, the effect of $\mathrm{M}$ receptor blockers in the treatment of myopia has been confirmed, and its representative drugs are mainly atropine, pirenzepine, racemic anisodamine and tropicamide. ${ }^{[9]}$ Atropine has been proposed as early as the 19th century. It is a non-selective muscarinic acetylcholine receptor antagonist, and it is widely believed to be effective in controlling myopia. ${ }^{[10]}$ Although atropine can delay the development of myopia, it has many side effects, such as dilated pupils, photophobia, and near vision disorders after use, and long-term use will reduce the efficacy of the drug. In addition, myopia rebound phenomenon will occur after stopping the drug. ${ }^{[11]}$

Acupuncture is a safe and effective treatment method, and it is gradually being accepted globally. Previously, studies have shown that acupuncture has a positive effect on slowing down the development of myopia. ${ }^{[12]}$ The research team repeatedly checked the literature and found that plum-blossom needle has a certain effect on the clinical efficacy of treating adolescent myopia and alleviating the development of myopia.This article aims to explore the effect of plum-blossom needles in the treatment of myopia, and hopes to further explore the best clinical diagnosis and treatment methods in the acupuncture category. At the same time, it is hoped that on this basis, the best treatment method can be improved or combined with other related treatment methods to provide better evidence for the clinical treatment of myopia.However, at present there exists no systematic review or study protocol published on this question. We thus have a opportunity to evaluate the issue and conceive this systematic review to determine the effectiveness and safety of plum-blossom needles for patients with myopia based on the most comprehensive and up-to-date resources.

\section{Methods}




\subsection{Study registration}

INPLASY systematical review protocol registration number is Inplasy protocol 202080026 on 08 August 2020 .https://inplasy.com/inplasy-2020-8-0026/).

This protocol will base on the preferred reporting items for systematic reviews and meta-analyses protocols (PRISMA-P) statement guidelines. ${ }^{[13]}$

\subsection{Inclusion criteria for study selection}

\subsubsection{Types of studies.}

All the RCTs of plum-blossom needle as the treatment for myopia will be involved in the review and no language or publication status limitation will be imposed. Non-rcts, review studies, case reports and animal experiments will be excluded

\subsubsection{Types of participants.}

Adolescents 18 years of age or younger who meet the diagnostic criteria for myopia冈and the included patients are simple myopia with a diopter of $-3.00 \mathrm{D}$ and below, with corrected visual acuity $\geq 1.0$.

Exclusion criteria $\triangle 1$. Those with other eye diseases 2 . Children with mentally handicapped and mental disorders 3. Those who are participating in Chinese and Western medicine or other treatment methods such as helium-neon laser therapy, visual physiological stimulation (CAM), massage, ear acupoints, etc.

\subsubsection{Types of interventions.}

Research using plum-blossom needle or combined with other therapies, without limiting the treatment time and dose.

\subsubsection{Types of outcome measures.}

Our primary outcomes will regard myopia progression and axial elongation as efficacy criteria. Myopia progression will be assessed as mean change in refractive error, measured in diopter, per year. Mean change in axial length, measured in millimeters, per year, will also be evaluated.

\subsubsection{Primary outcomes.}

1. Visual acuity score before and after treatment

2. Diopter 


\subsubsection{Secondary outcomes.}

1. Corneal curvature

2. Lens thickness

3. Ciliary body thickness

\subsection{Search methods for the identification of studies.}

\subsubsection{Electronic searches.}

Using the three keywords of "myopia, acupuncture, and plum blossom needle", from the establishment of the database to July 2020, the following electronic databases will be searched regardless of their status and language: Medline, EMBASE, Web of Science, the Cochrane Library, PubMed, China National Knowledge Infrastructure (CNKI), China Biology Medicine (CBM), Chinese Scientific Journal Database (VIP), Wanfang Database, Chinese Biomedical Literature Database (CBLD), Chinese Science and Technology Periodical Database (CSTPD). RCT registration websites, including http://www.ClinicalTrials.gov and http://www.chictr.org.cn, will also be searched. Review Manager V.5.4 will be used for statistical analysis. Two reviewers will independently select studies, extract and code the data, assess risk of bias of the included studies, and evaluate the quality of evidence for outcomes.

\subsubsection{Other searches.}

Taking account of possible omission, not only the published studies in journals but also gray literatures will be retrieved, mainly through conference papers and references.

\subsection{Data collection and analysis}

\subsubsection{Selection of studies.}

We plan to conduct this systematic review between 30 October 2020 and 30 July 2022. All reviewers have undergone a training to ensure a basic understanding of the background and purpose of the review. The selection of studies will be accomplished by two researches independently and be cross-checked. For literature management, EndnoteX9 will be utilized, with which the collected literatures will be imported and the duplicate ones will be deleted. Firstly, researchers will screen the titles and abstracts of the articles and exclude the distinctly ineligible ones. Afterward, the full text will be attentively inspected according to the inclusion and exclusion criteria previously set up. A third researcher will take part in the 
discussion and make the arbitration where there are discrepancies. The whole selection process will be presented in a PRISMA flow diagram (Fig. 1).

\subsubsection{Data extraction and management.}

Using a predefined data collection form, two researchers will complete the data

extraction. Extracted data shall include but not be limited to the following items: title, first author, publication time, sample size, severity and duration of myopia, age and gender of participants, outcomes, and adverse events. Inconsistencies will be resolved through discussion and consultation with a third researcher.

\subsubsection{Coping with the questionable data.}

We will try to contact the first or corresponding authors of the included studies by telephone or email to retrieve missing or insufficient trial data. If missing data are unavailable, we will make an assumption using the terms "missing at random" and

"not missing at random" to represent different scenarios. For the data "missing at random", only the available data will be analyzed. For the data "not missing at random", we will displace the missing data with replacement values and a sensitivity analysis will be used to determine whether the results are inconsistent.

\subsubsection{Assessment of risk of bias in included studies.}

Two reviewers will separately appraise the risk of bias of the involved studies according to Cochrane Handbook for Systematic Reviews of Intervention. There will be three levels of evaluation results utilizing three scores of " $\mathrm{L}$, , " $\mathrm{U}$," and " $\mathrm{H}$," respectively, indicating low-risk, uncertain, and high-risk. In the appraisal, seven sectors will be assessed, including random sequence generation, allocation concealment, blinding of participants and personnel, blinding of outcome assessment, completeness of outcome data, selective reporting, and other sources of bias. Discussion with a third reviewer will be conducted to cope with potential divergence, and the corresponding author will be connected when needed.

\subsubsection{Measures of treatment effect.}

To appraise the treatment effect for continuous data, mean difference (MD) in terms of $95 \%$ confidence interval $(\mathrm{Cl})$ will be applied, just as relative risk (RR) for dichotomous data.

\subsubsection{Assessment of heterogeneity.}


On the basis of the data analysis, random effect or fixed effect models will be employed according to the heterogeneity given by $R$ statistic value. To be concrete, a fixed effect model will be adopted if the heterogeneity is indicated as high $\left(R^{2}<50 \%\right)$; otherwise, a random effect model will be applied on the contrary.

\subsubsection{Assessment of reporting bias.}

The reporting bias will be visually indicated by funnel plots when studies are sufficient (at least 10 RCTs). If asymmetry is shown, Begg's and Egger's test will be completed and value of $P>0.05$ will be interpreted as no substantial reporting bias. Since the asymmetry of the funnel plot is not a substitute to publication bias, the potential reasons for it will be discriminated with terms like small sample size, low

methodological quality, or true heterogeneity.

\subsubsection{Data synthesis.}

The systematic review will be conducted with the use of RevMan 5.4. Taking account of the heterogeneity assessment, MD or RR with fixed or random effect model will be computed. Additionally, if heterogeneity is considered significant, the sensitivity or subgroup analysis will be generated to distinguish the source of it. When it comes to the situation that the data are insufficient for quantitative analysis, the review will only represent and summarize the evidence.

\subsubsection{Sensitivity analysis.}

As is mentioned above, sensitivity analysis will be done when the heterogeneity is greater than $50 \%$. Concretely, the meta-analysis will be reconducted after the studies with low quality and small sample size are excluded to identify whether these factors influence the result.

\subsubsection{Subgroup analysis.}

Subgroup analysis will also be performed after substantial heterogeneity is observed to find out the reasons. Characteristics like outcome type, disease duration, study quality, race, and so forth will be the content of the subgroup analysis.

\subsubsection{Quality of evidence.}

The Grading of Recommendations Assessment, Development and Evaluation (GRADE) working group methodology will be applied for the quality of evidence for all outcomes. ${ }^{[14]}$ Six domains will be assessed, containing risk of bias, consistency, 
directness, precision, publication bias and additional points. The assessments will be categorized into 4 levels: high, moderate, low, or very low.

\section{Discussion}

In recent years, with the improvement of people's living standards, the incidence of refractive errors has remained high. At present, the incidence of myopia in my country has risen from the fourth in the world in 1988 to the first in the world. ${ }^{[15]}$ In particular, the myopia of young people is increasing year by year, showing a trend of younger age. ${ }^{[16]} \mid \mathrm{t}$ is reported that the incidence of myopia in adolescents accounts for more than half of the total population, and the incidence of myopia in adolescents over 14 years old is as high as $80 \%$. This disease has become an important medical research hotspot. ${ }^{[17]}$ Some scholars have done more theoretical research on this disease, and believe that it is the result of multiple factors of genetics, diet, environment, and lifestyle. ${ }^{[18]}$ Timely prevention and treatment is an important means to improve the prognosis of this disease.Modern Western medicine has many treatment methods for this disease. ${ }^{[19,20]}$ Drugs such as anisodamine and atropine can relieve smooth muscle spasm. Although they have a certain effect on the control of myopia progression, there are still certain shortcomings. The degree of myopia is still slow growth, easy to repeat once the drug is stopped.The acceptance of refractive surgery and the treatment of wearing optical lenses is low, and the control effect of patients with myopia is unstable, and it has a greater impact on daily life and study.

In recent years, with the development of comprehensive medical thinking, some scholars have suggested that myopia adolescents need early prevention and treatment, and Chinese medicine has become an important part of the treatment of this disease. ${ }^{[21]}$ Modern Chinese medicine found that the pathological constitution of myopic adolescents is related to poor Qi and blood . ${ }^{[22]}$ Timely adjustment helps to regulate the function of the viscera, and stimulates the relevant acupoints to clear the meridians and run Qi and blood.TCM plum-blossom needle therapy is of great significance to the recovery of eye diseases, and clinical studies have confirmed that it is indeed effective. ${ }^{[23]}$ Therefore, we hope to systematically evaluate the efficacy and safety of plum-blossom needle in the treatment of adolescent myopia, and provide a better basis for clinical decision-making.

\section{Abbreviations}

MD: mean difference; PRISMA-P: preferred reporting items for systematic reviews and meta-analyses protocols; RCTs : randomized controlled trials; Cl: confidence interval ; RR: relative risk; SMD: standard mean difference; TCM: traditional Chinese medicine; WMD: weight mean difference.

\section{Declarations}

\section{Ethics approval and consent to participate}


Not applicable

\section{Consent for publication}

Not applicable

\section{Availability of data and materials}

Not applicable

\section{Competing interests}

The authors declare that they have no competing interests

\section{Funding}

This work was supported by Project of Jiangxi University of Traditional Chinese Medicine 1050 youth talent project (Grant number:5141900101), and Natural Science Youth Foundation Key Projects of Jiangxi Province (Grant No. 20192ACB21007).

\section{Authors' contributions}

Conceptualization: Siyuan Zhu, Jun Xiong.

Data curation: Siyuan Zhu, Lunbin Lu,Jun Chen,Genhua Tang,Xingchen Zhou, Zhiying Zhong,Han Guo.

Formal analysis: Siyuan Zhu,.Jun Chen

Investigation: Jun Xiong,Genhua Tang,Zhiying Zhong,Hao Fan

Methodology: Siyuan Zhu, Lunbin Lu,Jun Chen.

Software: Siyuan Zhu, Jun Chen

Supervision: Jun Xiong, Lunbin Lu,Hao Fan

Writing-original draft: Jun Xiong,Siyuan Zhu

Writing -review and editing: Jun Xiong, Genhua Tang, Lunbin Lu,Jun Chen.Hao Fan

Siyuan Zhu is the guarantor of this protocol. All authors read and approved the final manuscript. 


\section{Acknowledgements}

Not applicable

\section{References}

1. Dolgin E. The myopia boom. Nature. 2015;519(7543):276-8. doi:10.1038/519276a.

2. Morgan IG, Ohno-Matsui K, Saw SM. Myopia Lancet. 2012;379(9827):1739-48.

3. Williams KM, Verhoeven VJM, Cumberland P. Prevalence of refractive error in Europe: the european eye epidemiology (E3) Consortium. Eur J Epidemiol. 2015;30:305-15.

4. Holden BA, Fricke TR, Wilson DA, et al. Global Prevalence of Myopia and High Myopia and Temporal Trends from 2000 through 2050. Ophthalmology. 2016;123(5):1036-42.

5. Vitale S, Sperduto RD, Ferris FL. Increased prevalence of myopia in the United States between 19711972 and 1999-2004. Arch Ophthalmol. 2009;127:1632-9.

6. Wu PC, Huang HM, Yu HJ, Fang PC, Chen CT. Epidemiology of Myopia. Asia Pac J Ophthalmol (Phila). 2016;5(6):386-93.

7. Zhang Xin. Pay attention to the prevention and control of environmental risk factors for myopia in children and adolescents[J]. Chinese School Health,2018,39(01):6-8 + 12.

8. Li Y,Wang Zhiqiang,Wei Dong. Biological intervention effect of electric plum-blossom needle stimulation on adolescents with myopia[J]. Chinese Acupuncture and Moxibustion,2007(10):725728.

9. Ying $Y$, Xingtao Z. Experimental study of $M$ blockers delaying the progression of myopia. Chinese Journal of Ophthalmology Otolaryngology. 2010;10(5):334-5.

10. Saw SM, Shih-Yen EC, Koh A,et al. Interventions to retard myopia progression in children: an evidence based update. Ophthalmology. 2002;109(3):415i1/4ه421.

11. Chia A, Chua WH,Wen L,et al. Atropine for the treatment of childhood myopia: changes after stopping atropine $0.01 \%, 0.1 \%$ and0. 5\%Am J Ophthalmol. 2014;157(2):451-7.

12. Wei ML, Liu JP, Li N, Liu M. Acupuncture for slowing the progression of myopia in children and adolescents. Cochrane Database Syst Rev. 2011;(9):CD007842. Published 2011 Sep 7.

13. Shamseer $L$, Moher $D$, Clarke $M$, et al. Preferred reporting items for systematic review and metaanalysis protocols (PRISMA-P) 2015: elaboration and explanation. BMJ. 2015;350:1-25. Published 2015 Jan 2.

14. The GRADE Working Group. List of GRADE working group publications and grants. Available at: http://www.gradeworkinggroup. org/publications/index.htm.

15. Ophthalmology Branch of Chinese Medical Association. Expert consensus on children's refractive correction (2017) [J]. Chinese Journal of Optometry Vision Science. 2017;19(12):705-10.

16. Smaldone G, Campagna O, Pacella F. Computer use and onset of myopia in children\&58; a systematic review[J]. Senses \& Sciences, 2015, 2(1):1-7. 
17. Yang Linjuan Z, Xiaoling Wu. Jie. Progress in the prevention and control of adolescent myopia[J]. Chinese Journal of Maternal Child Health Research. 2017;28(12):1774-7.

18. Alifu KL, Lin D. Research progress on the risk factors of myopia[J]. New Advances in Ophthalmology. 2018;38(10):901-4.

19. Chen, Yanxian, He Mingguang. The prevention and treatment of myopia: from research evidence to clinical practice[J]. Chinese Journal of Optometry Vision Science. 2017;19(1):1-3.

20. Xu Jingjing T, Chunwen J, Wanqing, et al. Correlative research on imaging quality, refractive and structural parameters of school-age children with low myopia[J]. Chinese Journal of Optometry Vision Science. 2018;20(6):350.

21. He Qinqin Z, Biyu C, Bo, et al. Clinical application and mechanism analysis of Mingmuyanzhenfang in the treatment of eye diseases[J]. Liaoning Journal of Traditional Chinese Medicine. 2016;43(8):1726-7.

22. Hui Lv, Liping W, Fengren S, et al. Observation on the clinical efficacy of abdominal acupuncture in the treatment of myopia in children[J]. Chinese Journal of Acupuncture Moxibustion. 2015;35(6):567-70.

23. Zhang Shoukang D, Xiaohui Z, Lixia Z, Xiaojun D, Huifang W, Zhiqiang. Multi-center clinical observation of electric plum-blossom acupuncture treatment of adolescent myopia[J]. Chinese Journal of Ophthalmology of Traditional Chinese Medicine. 2011;21(02):74-7.

\section{Figures}




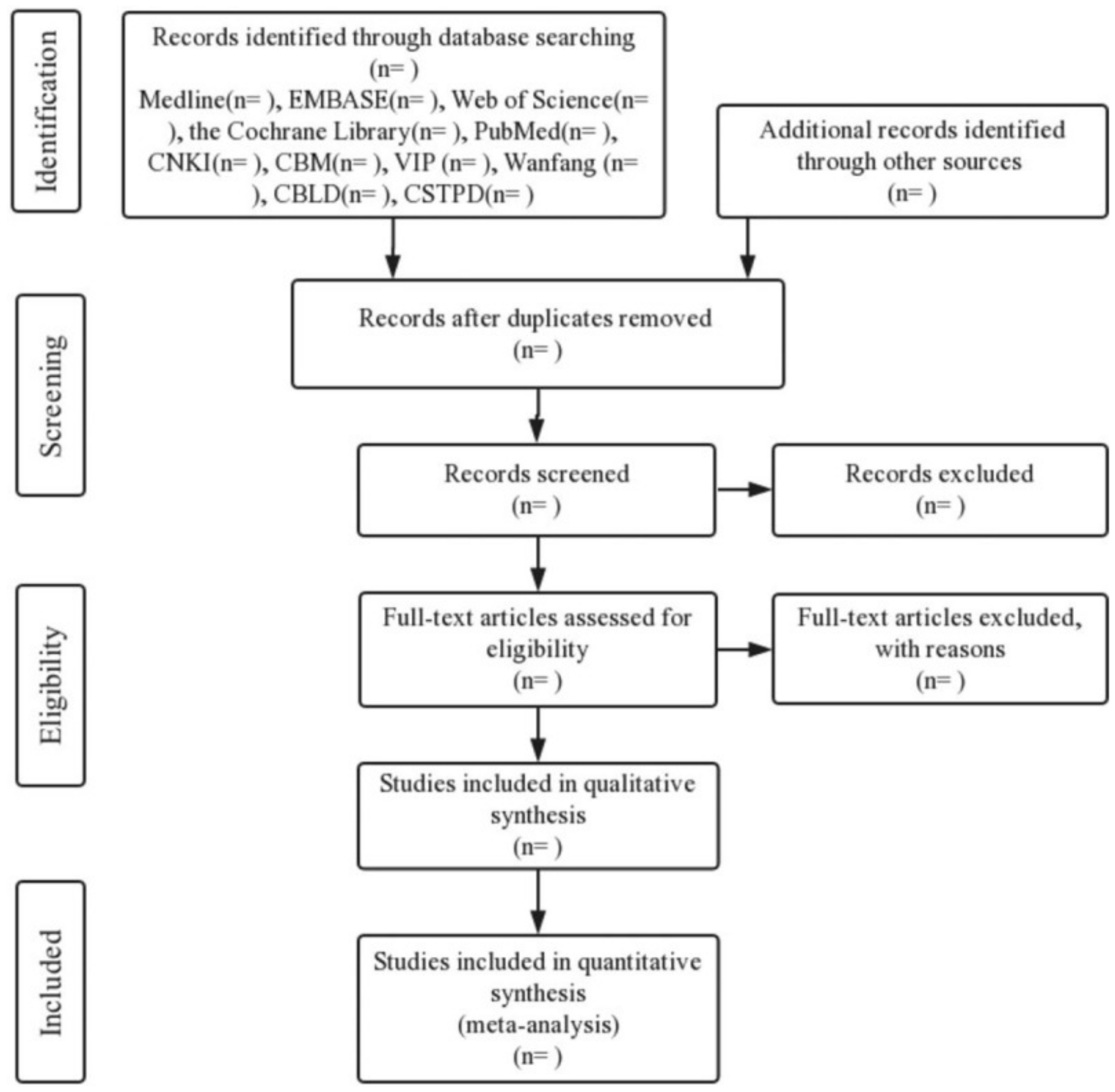

Figure 1

Flowchart of literature selection.

\section{Supplementary Files}

This is a list of supplementary files associated with this preprint. Click to download.

- PRISMAPchecklist.docx 